Diabetologe 2011 · 7:379-380

DOI 10.1007/s11428-011-0793-x

(c) Springer-Verlag 2011

\author{
J. Becker \\ Poliklinik für Zahnärztliche Chirurgie und Aufnahme, Westdeutsche Kieferklinik, \\ Universitätsklinikum Düsseldorf
}

\title{
Zahnfleischerkrankungen und Zuckerstoffwechsel
}

In dem Beitrag der Autoren Oesterreich $\mathrm{u}$. Ziller wird ein sehr interessanter Überblick über die aktuelle und zukünftige Mundgesundheitssituation auf der Basis aktueller epidemiologischer Daten gegeben. Die Autoren leiten daraus Schlussfolgerungen $a b$, welche Informationen der Zahnarzt über allgemeinmedizinische Erkrankungen haben muss, um seine $\mathrm{Pa}$ tienten entsprechend behandeln zu können. deutung zu. Diese Erkrankung ist für den Zahnarzt unter zahlreichen Gesichtspunkten von besonderer Bedeutung. Eine Information des Zahnarztes über Form und Schweregrad hilft ihm, seine Behandlung auf den Patienten besser abzustimmen, um z. B. unnötige Wartezeiten $\mathrm{zu}$ vermeiden, sowie anstrengendere und längere Behandlungen vorab mit dem Hausarzt abzustimmen, um den Blutzuckerspiegel des Patienten nicht unnötig zu beeinflussen.

Aktuelle Forschungsergebnisse haben weiterhin gezeigt, dass der Diabetes auch ein Risikofaktor für zahlreiche orale Erkrankungen ist, die bei Diabetikern häufiger und in höherem Schweregrad auftreten. Deshalb ist es für den Zahnarzt so bedeutsam, Risikopatienten zu identifizieren, sie einer abgestimmten Behandlung und einem speziellen „recall“ zuzuführen.

Insbesondere für parodontale Erkrankungen konnte in den letzten Jahren gezeigt werden, dass es eine enge Wechselwirkung zwischen der Kontrolle des Blutzuckers und oralen Entzündungen gibt. So begünstigt ein Diabetes die Entstehung und die Progression einer Parodontitis; eine Parodontitis erschwert zusätzlich die glykämische Kontrolle der Erkrankung.

\section{(D) Zahlreiche orale Erkrankungen treten bei Diabetikern häufiger und in höherem Schweregrad auf}

Der nachfolgende Beitrag von Deschner et al. stellt die wissenschaftlichen Studien vor, die heute zur Wechselbeziehung zwischen Zahnfleischerkrankungen und Zuckerstoffwechsel beim Diabetiker vorliegen. Ohne begleitende zahnärztliche Therapie ist es für den Diabetologen bei $\mathrm{Pa}$ tienten mit Zahnfleischerkrankungen komplizierter und ggf. auch nur eingeschränkter möglich, den Zuckerstoffwechsel erfolgreich zu kontrollieren.

Der dritte Beitrag der Autoren Sahm et al. beschäftigt sich mit dem Thema der Versorgung mit zahnärztlichen Implantaten. Implantate haben heute auf der Grundlage einer sehr guten wissenschaftlichen Basis einen unverzichtbaren Stellenwert bei der prothetischen Versorgung der Bevölkerung. Es wird geschätzt, dass derzeit jährlich ca. 1 Mio. zahnärztlicher Implantate inseriert werden. taten gestützten prothetischen Versorgung muss jedoch berücksichtigt werden, dass
Für den Langzeiterfolg der auf Implan- auch an Implantaten Zahnfleischentzündungen auftreten können und dass diese dann einen komplizierteren und schwerer zu behandelnden Verlauf nehmen können. In dem Beitrag wird die aktuelle wissenschaftliche Datenlage beleuchtet, und es werden die speziellen Zahnfleischerkrankungen bei Implantaten und deren Therapieoptionen ausführlich vorgestellt.

Die Beiträge in diesem Heft von Der Diabetologe sollen dazu beitragen, das gegenseitige Verständnis zwischen Endokrinologie und Zahnmedizin zum Vorteil der Patienten zu vertiefen.

Ihr

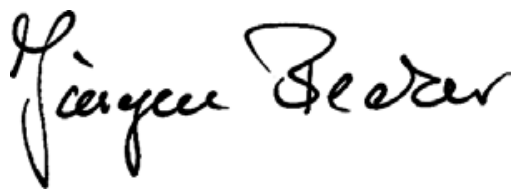

J. Becker

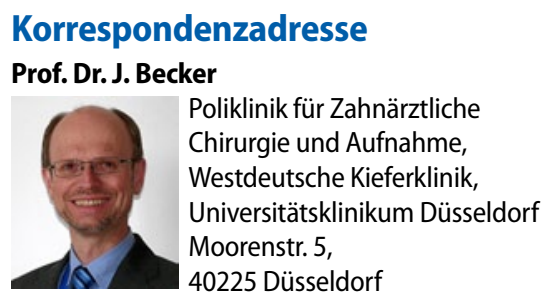




\section{Hier steht eine Anzeige.}

Springer 